

Available online at https://www.irjst.com/

International Research Journal of Science and Technology

ISSN: 2707-3955

DOI: https://doi.org/10.46378/irjst.2020.010311

\title{
Application Of Remote Sensing And GIS in the Identification of Waste Land in Gadilam Lower Sub Basin of Ponnaiyar Basin in Cuddalore District.
}

\author{
S. Deeba ${ }^{1}$, R.S. Soundharya ${ }^{2}$, D. Radhakrishnan ${ }^{3 *}$ \\ ${ }^{1}$ Department of Civil Engineering, Tagore Engineering College, Chennai. \\ ${ }^{2}$ PG Scholar, Water Resources Engineering and Management, Faculty of Engineering and Technology, Annamalai \\ University, Chidambaram, Tamil Nadu. \\ ${ }^{3}$ Water Resources and Irrigation Engineering, Water Technology Institute, Arba Minch University, Ethiopia.
}

\begin{tabular}{llll}
\multicolumn{2}{l}{ Paper Status } & & \\
Received : & Apr 2020 \\
Accepted & $:$ & May 2020 \\
Published & $:$ & Jun 2020
\end{tabular}

Key Words

GIS

IRS

LISS

Remote Sensing

Waste Land

\section{Abstract}

Owing to increasing pressure of population there is an excessive demand of lands both in agriculture and non-agriculture purposes. This has resulted in vast stretches of waste land such as degraded land, soil salinity, water logging, desertification, soil erosion etc.; decrease in per capita cultivable land beside ecological imbalances. The pilot studies were carried out by NRSA and evaluated by different experts, and it was decided that the waste land maps on 1:50000 scales depicting the village boundaries were considered to be adequate for reclamation purpose. At present, the new techniques like remote sensing and GIS are used to delineate waste lands, faster and also produce better results. Current study, choose the study area of Gadilam river sub basin falling in Cuddalore and Villupuram districts. Gadilam is one of the sub-basin of Ponnaiyar basins. Main goal of the study examined the role of remote sensing and GIS for the identification of wasteland and to convert the wasteland into fertile land. Study conducted, and various data collected at different levels related to rainfall, control well details, water quality etc. Satellite imagery of the study area is also collected and interpreted. Furthermore, the maps like geology, soil are also collected. Geomorphology, land use, lineament, waste land were generated from satellite imagery. By overlaying the various thematic maps, wastelands and wasteland categories are identified. With the help of ground water wastelands of this study area is to be converted into fertile land.

Copyright (C) 2020: S. Deeba, R.S. Soundharya, D. Radhakrishnan. This is an open access distribution, and reproduction in any medium, provided Access article distributed under the Creative Commons Attribution License the original work is properly cited License, which permits unrestricted use.

Citation: S. Deeba, R.S. Soundharya, D. Radhakrishnan. “Application Of Remote Sensing And GIS in the Identification of Waste Land in Gadilam Lower Sub Basin of Ponnaiyar Basin in Cuddalore District.”.International Research Journal of Science and Technology, $1(3), 256-261,2020$.

\section{Introduction}

Identification of waste land is an important resource and can hold key to the economics of any country. In India various projects have been implemented to convert the waste lands into fertile land. Now a days waste lands increased due to industrial and modern agricultural development In India various projects have been implemented to convert the waste lands into fertile land.

\footnotetext{
* Corresponding Author: D.Radhakrishnan

Water Resources and Irrigation Engineering, Water Technology Institute, Arba Minch University, Ethiopia.

Email: radhadgeo@gmail.com
}

The small scale and low resolution of data could not be mapped, the land less than100 hectares. Moreover, the small scale maps could not be used for massive reclamation at micro-level. Therefore NWDB decided to map the different categories of waste lands on a large scale (1:50000) which could enable the identification of the waste land. Identification of waste land is an important resource and can hold key to the economics of any country $[1,2]$.

Waste land is described as "degraded land which can be brought under vegetative cover with reasonable effort and which is currently on utilized and which is 
deteriorating for lack of appropriate water and soil management or on account of natural causes. Waste land can result from inherent/ imposed disabilities section by location, environment, Chemical and physical properties of the soil of financial or management constraints". Waste lands therefore need to be mapped primarily to identify them so as to prepare a proper planning to convert them into productive lands.

The Landsat Thematic mapper (TM) satellite images were used to prepare a detailed wasteland maps on 1:50000 scale in the period between 1986 and 1988 Indian Remote sensing satellite (IRS) LISS II and LISS III data during 1990-1997. The realization of the [problems pertaining to wasteland management have initiated the formulation of nationwide data base (1:50000 scale) by NRSA, integrating remote sensing and GIS techniques (NRSA 1997) which helped to device methods to solve the problems of waste lands and suggest reclamation measures [3-5]. Gupta et al. [6] the application of remote sensing in inventorying degraded lands is emphasized by visually delineating various land units such as agriculture, forest, open scrubs erosional surfaces, kankars and saline areas using IRS -1A LISS II data. Nagarathinam, 1997 [7] explained the usefulness of remote sensing and GIS based approach to assess land potential for waste land development at village level in a study conducted around Kalluthu village, Usilampatti block of Tamilnadu, India. Pradeep Kumar, 2001 [8] integrated various thematic layers such as lithology, landforms, soil, groundwater level, rainfall and slope to understand their influences on waste lands and also explained the utility of remote sensing and GIS techniques for reclaiming them.

\subsection{Waste Land Classification System}

Different agencies including data thrown up by latest technological tools like remote sensing it became evident that a precise definition of waste lands of various categories is required. Therefore planning commission and National waste lands development board (NWDB) to arrive at precise definition of categories constituted a technical task force group. The classification system developed by the technical task force group and subsequently modified into 13 categories of waste lands. They are as follows:

1) Gullied /or ravenous land.

2) Land with or without scrub.

3) Waterlogged and marshy land.

4) Land affected by salinity.

5) Shifting cultivation area.

6) Underutilized /degraded notified forest land.

7) Degraded pastures

8) Degraded land under plantation.

9) Sands -Deserts.
10) Mining /industrial waste lands.

11) Barren rocky /stony waste.

12) Steep sloping area.

13) Snow covered area.

In general the waste land categorized by the government of India as in state wise and district wise of Tamil Nadu, India is shown in Tables 1 and 2.

\section{Methodology}

\subsection{Study Area}

The study area situated in a part of Gadilam lower sub basin (Figure 1) comes under Villupuram and Cuddalore district, Tamilnadu.The area located in the topo sheets are 58/M5, M6, M9, M10, M13, published by survey of India in the year 2003 of 1:50000 scale lies between the latitudes and longitudes 79'40" \& 79'35", 11'25" \& 11'30". Major settlements roads, railway lines, tanks, man-made canals river Gadilam is marked in the study area map. The Gadilam sub basin covers about $335.78 \mathrm{sq} \mathrm{km}$. The Gadilam is about $178.35 \mathrm{~km}$ which flows from west to east and merges with Bay of Bengal $[9,10]$.

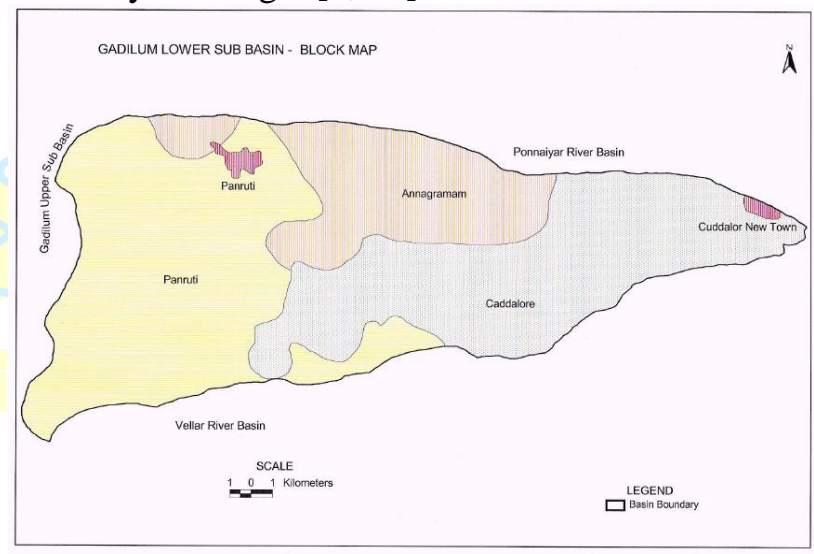

Figure 1. Gadilam Lower Sub basin, Cuddalore block map

The study was followed in waste land identification by employing satellite data. Data collected from Satellite IRS IC, LISS III Panchromatic through Institute of remote sensing, Chennai, Tamil Nadu (India), and were analyzed in two ways as [11]:

1. Digital analysis and

2. Visual analysis

Visual analysis involves the interpretation based on image characteristics such as tone, colour, texture, pattern, shape, size, location and association to identify and delineate different types of waste lands. The final thematic details and the village boundaries were transferred to base map prepared using survey of India; each waste lands map contains waste land information from satellite data, village boundaries from revenue maps and base details from SOI topo sheets. The district wise reports explaining the distribution of waste lands including the general information about the rainfall, temperature, soils etc. were prepared and distributed to the users. 
Table 1. State wise waste land category in India

\begin{tabular}{|c|c|c|c|c|c|c|c|c|c|c|c|c|c|c|c|c|}
\hline States & 1 & 2 & 3 & 4 & 5 & 6 & 7 & 8 & 9 & 10 & 11 & 12 & 13 & 14 & 15 & 16 \\
\hline 1.AP & 692.68 & 20256.64 & 1035.02 & 603.26 & 13.80 & \begin{tabular}{|l|}
22237.78 \\
\end{tabular} & 709.29 & 52.91 & 464.70 & 98.88 & \begin{tabular}{|l|l|}
5196.27 \\
\end{tabular} & 388.96 & 0.00 & 51750.19 & \begin{tabular}{|l|}
275068.00 \\
\end{tabular} & 18.81 \\
\hline 2.Aruna & 0.00 & 3326.78 & 41.47 & 0.00 & 3088.08 & 1416.67 & 2134.99 & 6.07 & \begin{tabular}{|l|}
309.43 \\
\end{tabular} & 0.30 & 1262.36 & \begin{tabular}{|l}
7.93 \\
\end{tabular} & 6732.25 & 18326.25 & \begin{tabular}{|l|}
83743.00 \\
\end{tabular} & 21.88 \\
\hline 3.Assam & 0.00 & 843,72 & 1633.56 & 0.00 & 8391.48 & 3112.71 & 2217.85 & 0.00 & \begin{tabular}{|l|}
3764.54 \\
\end{tabular} & 0.43 & 54.88 & 0.00 & 0.00 & 20019.17 & 78438.00 & 25.52 \\
\hline 4.Bihar & 559.17 & 4689.93 & \begin{tabular}{|l|l|}
1198.87 \\
\end{tabular} & 0.51 & 45.45 & 13066.53 & 184.97 & 79.80 & 222.08 & 184.23 & \begin{tabular}{|l|}
688.91 \\
\end{tabular} & \begin{tabular}{|l|l|}
97.10 \\
\end{tabular} & 0.00 & 20997.55 & \begin{tabular}{|l|l|}
173877.00 \\
\end{tabular} & 12.08 \\
\hline 5.Goa & 0.00 & 292.83 & 41.02 & 0.00 & 0.00 & 71.99 & 2.47 & 32.19 & 0.00 & 110.73 & 58.55 & 3.49 & 0.00 & 613.27 & 3702.00 & 16.57 \\
\hline 6.Gujarat & 1013.39 & 21786.72 & 2656.28 & 7637.34 & 0.00 & 5443.02 & 387.45 & 78.32 & \begin{tabular}{|l|}
188.42 \\
\end{tabular} & 49.66 & \begin{tabular}{|l|l|}
3293.39 \\
\end{tabular} & \begin{tabular}{|l|}
487.31 \\
\end{tabular} & 0.00 & 43021.28 & \begin{tabular}{|l|l|}
196024.00 \\
\end{tabular} & 21.95 \\
\hline 7.Haryana & 49,50 & 988.42 & \begin{tabular}{|l|}
238.30 \\
\end{tabular} & 285.63 & 0.00 & \begin{tabular}{|l|}
732.52 \\
\end{tabular} & 721.65 & 134.12 & 465.01 & 13.72 & 105.12 & 0.00 & 0.00 & 3733.98 & \begin{tabular}{|l|l}
44212.00 \\
\end{tabular} & 8.45 \\
\hline 8.HP & 121.89 & 2056.50 & 15.69 & 1.36 & 0.00 & 4589.98 & 4278.17 & \begin{tabular}{|l|l|}
2457.59 \\
\end{tabular} & \begin{tabular}{|l|}
105.04 \\
\end{tabular} & 85.66 & 3858.04 & \begin{tabular}{|l|l|}
1529.67 \\
\end{tabular} & \begin{tabular}{|l|}
12559.42 \\
\end{tabular} & \begin{tabular}{|l|l|}
31659.00 \\
\end{tabular} & \begin{tabular}{|l|l|}
55673.00 \\
\end{tabular} & 56.87 \\
\hline 9.J\&K & 21.25 & 4495.30 & 246.60 & 0.00 & 0.00 & 2491.66 & 267.51 & 640.56 & 869.26 & 0.31 & \begin{tabular}{|l|}
32821.50 \\
\end{tabular} & \begin{tabular}{|l|}
1685.42 \\
\end{tabular} & \begin{tabular}{|l|}
21904.97 \\
\end{tabular} & \begin{tabular}{|l|l|}
65444.24 \\
\end{tabular} & \begin{tabular}{|l|l|}
101387.00 \\
\end{tabular} & 64.55 \\
\hline 10.Karnataka & 301.52 & 9087.68 & \begin{tabular}{|l|}
32.76 \\
\end{tabular} & 125.11 & 0.00 & 8299.41 & 97.48 & 104.74 & 43.96 & $\begin{array}{l}77.78 \\
\end{array}$ & \begin{tabular}{|l|}
2627.89 \\
\end{tabular} & \begin{tabular}{|l|}
40.97 \\
\end{tabular} & 0.00 & 20839.28 & \begin{tabular}{|l|}
191791.00 \\
\end{tabular} & 10.87 \\
\hline 11.Kerala & 0.00 & 357.93 & 138.00 & 0.00 & 0.00 & 809.30 & 3.99 & 25.65 & \begin{tabular}{|l|}
27.87 \\
\end{tabular} & \begin{tabular}{|l|}
0.49 \\
\end{tabular} & \begin{tabular}{|l|}
146.46 \\
\end{tabular} & 140.49 & 0.00 & 1448.18 & 38863.00 & 3.73 \\
\hline 12.MP & 7569.11 & 36977.87 & 51.72 & 162.81 & 0.00 & \begin{tabular}{|l|}
20437.77 \\
\end{tabular} & 302.44 & 910.40 & 24.57 & 141.44 & \begin{tabular}{|l|l|}
2950.97 \\
\end{tabular} & 184.65 & 0.00 & 69713.75 & \begin{tabular}{|l|l|}
44346.00 \\
\end{tabular} & 15.72 \\
\hline 13.Maharastra & 1700.37 & 31386.91 & \begin{tabular}{|l|}
527.57 \\
\end{tabular} & 251.66 & 0.00 & \begin{tabular}{|l|}
13430.67 \\
\end{tabular} & 1349.40 & \begin{tabular}{|l|}
687.43 \\
\end{tabular} & 77.63 & 100.45 & 2587.42 & \begin{tabular}{|l|}
1389.57 \\
\end{tabular} & 0.00 & 53489.08 & 307690.00 & 17.38 \\
\hline 14.Manipur & 0.00 & 1.32 & 324.60 & 0.00 & \begin{tabular}{|l|}
12014.06 \\
\end{tabular} & 608.64 & 0.00 & 0.00 & 0.00 & 0.00 & 0.00 & 0.00 & 0.00 & \begin{tabular}{|l|}
12948.62 \\
\end{tabular} & \begin{tabular}{|l|}
22327.00 \\
\end{tabular} & 58.00 \\
\hline 15.Megalaya & 0.00 & 4190.63 & \begin{tabular}{|l|l|}
14.87 \\
\end{tabular} & 0.00 & 2086.77 & 3612.11 & 0.00 & 0.00 & 0.00 & 0.00 & 0.00 & 0.00 & 0.00 & 9904.38 & \begin{tabular}{|l|}
22429.00 \\
\end{tabular} & 44.16 \\
\hline 16.Mizoram & 0.00 & 0.00 & \begin{tabular}{|l|l|}
0.00 \\
\end{tabular} & 0.00 & 3761.23 & 310.45 & 0.00 & 0.00 & 0.00 & 0.00 & 0.00 & 0.00 & 0.00 & 4071.68 & \begin{tabular}{|l|}
21081.00 \\
\end{tabular} & 19.31 \\
\hline 17.Nagaland & 0.00 & 1596.46 & 0.00 & 0.00 & 5224.65 & 1582.99 & 0.00 & 0.00 & 0.00 & 0.00 & 0.00 & 0.00 & 0.00 & 8404.10 & \begin{tabular}{|l|}
16579.00 \\
\end{tabular} & 50.69 \\
\hline 18.Orissa & 185.62 & 8358.68 & 379.10 & 51.49 & 115.28 & \begin{tabular}{|l|}
10014.07 \\
\end{tabular} & 13.43 & 193.93 & \begin{tabular}{|l|}
212.49 \\
\end{tabular} & 35.45 & \begin{tabular}{|l|}
1574.09 \\
\end{tabular} & 207.88 & 0.00 & 21341.71 & \begin{tabular}{|l|l|}
155707.00 \\
\end{tabular} & 13.71 \\
\hline 19.Punjab & 168.62 & 339.44 & 352.01 & 173.29 & 0.00 & 353.29 & 113.71 & 81.58 & \begin{tabular}{|l|}
619.67 \\
\end{tabular} & \begin{tabular}{|l|}
26.69 \\
\end{tabular} & \begin{tabular}{|l|}
0.00 \\
\end{tabular} & \begin{tabular}{|l|}
0.00 \\
\end{tabular} & 0.00 & 2228.40 & \begin{tabular}{|l|}
50362.00 \\
\end{tabular} & 4.42 \\
\hline 20.Rajasthan & 4952.77 & 27152.76 & 289.66 & 2722.99 & 0.00 & \begin{tabular}{|l|}
12541.89 \\
\end{tabular} & 12208.44 & 21.14 & \begin{tabular}{|l|}
40639.51 \\
\end{tabular} & 128.65 & \begin{tabular}{|l|l|}
4799.02 \\
\end{tabular} & 182.28 & 0.00 & 105639.00 & 342239.00 & 30.87 \\
\hline 21.Sikkim & 0.00 & 1073.11 & 0.00 & 0.00 & 0.00 & 1060.57 & 0.00 & 0.00 & \begin{tabular}{|l|}
0.00 \\
\end{tabular} & \begin{tabular}{|l|}
0.00 \\
\end{tabular} & 10.34 & \begin{tabular}{|l|}
0.00 \\
\end{tabular} & 1425.66 & 3569.68 & 7096.00 & 50.30 \\
\hline 22.Tripura & 0.00 & 286.87 & \begin{tabular}{|l|l|}
0.11 \\
\end{tabular} & 0.00 & 400.88 & 588.18 & 0.00 & 0.00 & 0.00 & 0.00 & 0.00 & 0.00 & 0.00 & 1276.03 & \begin{tabular}{|l|}
10486.00 \\
\end{tabular} & 12.17 \\
\hline $23 . \mathrm{TN}$ & 226,12 & 7697.91 & 415.80 & 2479.73 & 0.53 & 9634.25 & 168.94 & 221.96 & \begin{tabular}{|l|}
590.80 \\
\end{tabular} & \begin{tabular}{|l|}
120.48 \\
\end{tabular} & 1155.92 & \begin{tabular}{|l|}
301.50 \\
\end{tabular} & 0.00 & \begin{tabular}{|l|}
23013.90 \\
\end{tabular} & \begin{tabular}{|l|l}
130058.00 \\
\end{tabular} & 17.70 \\
\hline 24.UP & 2808.52 & 5498.99 & \begin{tabular}{|l|l|}
4981.43 \\
\end{tabular} & \begin{tabular}{|l|l|}
5811.94 \\
\end{tabular} & 0.00 & 3338.32 & 446.36 & 50.44 & \begin{tabular}{|l|}
470.21 \\
\end{tabular} & 29.26 & 1180.13 & \begin{tabular}{|l|}
992.83 \\
\end{tabular} & \begin{tabular}{|l|}
13166.37 \\
\end{tabular} & \begin{tabular}{|l|}
38772.80 \\
\end{tabular} & \begin{tabular}{|l|l|}
294411.00 \\
\end{tabular} & 13.17 \\
\hline 25.WB & 171.90 & 1245.16 & 1931.54 & 131.25 & 0.00 & \begin{tabular}{|l|}
777.58 \\
\end{tabular} & 384.97 & 2.93 & \begin{tabular}{|l|}
879.13 \\
\end{tabular} & 47.34 & \begin{tabular}{|l|}
130.46 \\
\end{tabular} & 16.24 & 0.00 & 5718.48 & \begin{tabular}{|l|}
88752.00 \\
\end{tabular} & 6.44 \\
\hline 26.Union Teri & 12.83 & 25.74 & 24.60 & 39.01 & 0.00 & 289.97 & 5.43 & 49.34 & 47.33 & 0.00 & 83.05 & 0.00 & 0.00 & 574.30 & \begin{tabular}{|l|}
10973.00 \\
\end{tabular} & 5.23 \\
\hline Total & 20553.35 & 194014.29 & 16568.45 & 20477.38 & 35142.20 & 140652.31 & \begin{tabular}{|l|}
25978.91 \\
\end{tabular} & 5828.09 & 50021.65 & 1252.13 & \begin{tabular}{|l|}
64584.77 \\
\end{tabular} & \begin{tabular}{|l|}
7656.29 \\
\end{tabular} & \begin{tabular}{|l|}
55788.49 \\
\end{tabular} & 638518.31 & 3166414.00 & 20.17 \\
\hline $\begin{array}{l}\text { 1. Gullied /or } r \\
\text { 2. Land with or } \\
\text { 3. Waterlogged } \\
\text { 4. Land affecte } \\
\text { 5. Shifting cult } \\
\text { Sources: } 1: 500 \\
\text { Note: } 1208490\end{array}$ & $\begin{array}{l}\text { venous lanc } \\
\text { without scr } \\
\text { and marshy } \\
\text { l by salinity } \\
\text { vation area. }\end{array}$ & b. & $\begin{array}{l}\text { Under-utili } \\
\text { 7. Degraded } \\
\text { 8. Degraded } \\
\text { Sands-Dese } \\
\text { 0. Mining /in }\end{array}$ & $\begin{array}{l}\text { ed /degrad } \\
\text { pastures } \\
\text { land under } \\
\text { ts. }\end{array}$ & d notified for & rest land. & $\begin{array}{l}\text { 1. Barren ro } \\
\text { 12. Steep sl } \\
\text { 13. Snow co } \\
\text { 4. Total was } \\
\text { 15. Total G }\end{array}$ & $\begin{array}{l}\text { ky /stony y } \\
\text { ping area. } \\
\text { vered area. } \\
\text { elands } \\
\text { og. Area }\end{array}$ & vaste. 16. & $\%$ of total & Geog. area & & & & & \\
\hline
\end{tabular}


Table 2. District wise waste land category in Tamil Nadu, India

\begin{tabular}{|c|c|c|c|c|c|c|c|c|c|c|c|c|c|c|c|c|c|}
\hline No & Districts & 1 & 2 & 3 & 4 & 5 & 6 & 7 & 8 & 9 & 10 & 11 & 12 & 13 & 14 & 15 & 16 \\
\hline 1 & Chennai & 0.00 & 0.89 & 0.00 & 1.02 & 0.00 & 2.47 & 0.00 & 0.00 & 4.25 & 0.00 & 0.00 & 0.00 & 0.00 & 8.63 & 171.00 & 5.05 \\
\hline 2 & Coimbatore & 0.62 & 371.81 & 0.00 & 299.03 & 0.00 & 991.79 & 0.00 & 0.00 & 0.28 & 8.01 & 39.13 & 42.34 & 0.00 & 1753.01 & 7468.00 & 23.47 \\
\hline 3 & Dharmapuri & 19.94 & 598.06 & 0.00 & 24.93 & 0.00 & 1463.73 & 6.73 & 4.30 & 0.00 & 0.00 & 311.14 & 3.18 & 0.00 & 2432.01 & 9642.00 & 25.22 \\
\hline 4 & Kanchipuram & 0.00 & 279.24 & 63.29 & 326.64 & 0.00 & 185.54 & 0.00 & 27.44 & 46.98 & 4.89 & 18.77 & 0.03 & 0.00 & 952.82 & 4433.00 & 21.49 \\
\hline 5 & Kanniayakumari & 4.37 & 72.79 & 0.00 & 0.00 & 0.00 & 95.00 & 0.00 & 4.40 & 12.41 & 0.00 & 1.18 & 26.37 & 0.00 & 216.52 & 1674.00 & 12.93 \\
\hline 6 & Madurai & 1.04 & 1050.29 & 0.00 & 20.56 & 0.00 & 710.66 & 0.00 & 14.28 & 38.76 & 0.00 & 168.81 & 78.71 & 0.00 & 2091.11 & 12899.0 & 16.21 \\
\hline 7 & Teni & \multicolumn{16}{|c|}{ The districts is newly formed out of the Madurai districts and the statistics included as in combined Madurai district } \\
\hline 8 & Dindugul & \multicolumn{16}{|c|}{ The districts is newly formed out of the Madurai districts and the statistics included as in combined Madurai district } \\
\hline 9 & Nagapattinam & 0.00 & 3.28 & 100.54 & 114.07 & 0.00 & 15.44 & 0.00 & 0.00 & 72.55 & 0.00 & 0.33 & 0.00 & 0.00 & 306.01 & 2718.00 & 11.26 \\
\hline 10 & Nilgiris & 0.00 & 9.57 & 0.00 & 0.00 & 0.00 & 835.57 & 0.00 & 1.01 & 0.00 & 0.00 & 3.72 & 12.30 & 0.00 & 862.17 & 2549.00 & 33.62 \\
\hline 11 & Vellore & 6.03 & 652.42 & 0.00 & 58.34 & 0.00 & 1430.13 & 0.18 & 30.40 & 6.89 & 0.51 & 157.61 & 15.70 & 0.00 & 2358.21 & 12268.0 & 19.22 \\
\hline 12 & Tiruvannamalai & \multicolumn{16}{|c|}{ The districts is newly formed out of the Vellore districts and the statistics included as in combined North Arcod district } \\
\hline 13 & Erode & 1.52 & 328.14 & 0.00 & 46.14 & 0.00 & 1614.75 & 157.44 & 6.17 & 1.30 & 0.19 & 35.26 & 10.80 & 0.00 & 2200.71 & 8165.00 & 26.95 \\
\hline 14 & Phudukkottai & 9.92 & 358.52 & 0.00 & 40.67 & 0.00 & 32.65 & 0.00 & 0.00 & 58.16 & 0.00 & 10.07 & 0.08 & 0.00 & 510.07 & 4664.00 & 10.94 \\
\hline 15 & Ramanadhapura & 7.15 & 393.28 & 10.39 & 18.67 & 0.00 & 87.36 & 0.58 & 0.73 & 80.92 & 3.51 & 64.61 & 0.00 & 0.00 & 667.20 & 12533.0 & 5.32 \\
\hline 16 & Virudhunagar & \multicolumn{16}{|c|}{ The districts is newly formed out of the Ramanathapuram districts and the statistics included as in combined Ramanathapuram district } \\
\hline 17 & Sivagangai & \multicolumn{16}{|c|}{ The districts is newly formed out of the Ramanathapuram districts and the statistics included as in combined Ramanathapuram district } \\
\hline 18 & Salem & 0.00 & 328.16 & 0.00 & 32.57 & 0.00 & 751.20 & 4.01 & 93.14 & 2.06 & 47.23 & 177.29 & 70.83 & 0.00 & 1306.49 & 8650.00 & 17.42 \\
\hline 19 & Namakkal & \multicolumn{16}{|c|}{ The districts is newly formed out of the Salem districts and the statistics included as in combined Salem district } \\
\hline 20 & Cuddalore & 24.13 & 750.99 & 57.07 & 213.87 & 0.53 & 265.95 & 0.00 & 20.18 & 23.07 & 33.25 & 42.26 & 2.70 & 0.00 & 1433.98 & 10900.0 & 13.16 \\
\hline 21 & Villupuram & \multicolumn{16}{|c|}{ The districts is newly formed out of the South Arcod districts and the statistics included as in combined South Arcod district } \\
\hline 22 & Thanjavur & 1.79 & 77.44 & 36.39 & 140.62 & 0.00 & 0.00 & 0.00 & 5.37 & 17.74 & 0.04 & 0.00 & 0.00 & 0.00 & 276.39 & 3397.00 & 8.14 \\
\hline 23 & Trichy & 145.47 & 677.28 & 0.00 & 8.05 & 0.00 & 434.91 & 0.00 & 0.40 & 50.39 & 2.70 & 68.96 & 1.34 & 0.00 & 1389.50 & 10991.0 & 12.64 \\
\hline 24 & Karur & \multicolumn{16}{|c|}{ The districts is newly formed out of the Trichirappalli districts and the statistics included as in combined Trichirappalli district } \\
\hline 25 & Perambalur & \multicolumn{16}{|c|}{ The districts is newly formed out of the Trichirappalli districts and the statistics included as in combined Trichirappalli district } \\
\hline 26 & Thirunelveli & 3.97 & 955.27 & 0.00 & 503.99 & 0.00 & 533.80 & 0.00 & 0.13 & 40.78 & 11.28 & 43.95 & 36.55 & 0.00 & 2129.72 & 6824.00 & 31.21 \\
\hline 27 & Thiruvallur & 0.17 & 465.90 & 107.05 & 144.92 & 0.00 & 80.81 & 0.00 & 13.77 & 12.46 & 5.95 & 11.40 & 0.57 & 0.00 & 843.00 & 3423.00 & 24.63 \\
\hline 28 & Thiruvarur & 0.00 & 2.64 & 40.52 & 151.00 & 0.00 & 0.00 & 0.00 & 0.00 & 13.15 & 0.00 & 0.00 & 0.00 & 0.00 & 207.41 & 2098.00 & 9.89 \\
\hline 29 & Tuticorin & 0.00 & 314.94 & 0.45 & 334.65 & 0.00 & 102.49 & 0.00 & 1.24 & 108.85 & 2.90 & 3.43 & 0.00 & 0.00 & 868.95 & 4591.00 & 18.93 \\
\hline & Total & 226.12 & 7697.91 & 415.80 & 2479.74 & 0.53 & 9634.25 & 168.94 & 221.96 & 590.80 & 120.46 & 1165.92 & 301.50 & 0.00 & 23013.91 & 130058.00 & 347.90 \\
\hline
\end{tabular}


Table 3. Results of Waste lands in Gadilam lower sub basin found through Satellite image

\begin{tabular}{|l|l|l|l|l|}
\hline S1.No. & Name of villages & Waste land categories & $\begin{array}{l}\text { Total Area } \\
\text { sq km }\end{array}$ & \% of waste land \\
\hline 1. & Kondur & Sandy soil & 1.79 & 5 \\
\hline 2. & Eydanur & Sandy soil & 2.74 & 4 \\
\hline 3. & Karuppaditthundu & Gullied land deep & 2.75 & 13.8 \\
\hline 4. & Nadukuppam & Gullied land deep & 6.25 & 3.5 \\
\hline 5. & Thenkuthu & Gullied land shallow & 3.58 & 16.2 \\
\hline 6. & Vilangalpattu & Gullied land shallow & 5.41 & 8.7 \\
\hline 7. & Thirumanikuzhi & Barren land & 3.92 & 36.9 \\
\hline 8. & Padhirikkuppam & Barren land & 1.28 & 46.1 \\
\hline
\end{tabular}

\subsection{Instruments Used and Method of Interpretation}

Planimeter is an instrument to measure the area of the closed curve, length of the line; arc angle etc on the map. It is also called as computer co-coordinating curvimeter.

Computer and various GIS software are now used in data recording, analysis, image processing and interpretation of digital images. ARC/INFO, MAP/INFO and ERDAS are the common GIS software used for data analysis and image processing.

\subsection{Satellite Data Analysis and Interpretation}

Remote sensing is a science and technology and art of obtaining reliable information about an object, without being physical contact with the object the data analysis examining the data using various viewing and interpretation devices to analysis pictorial data and /or a computer analysis digital sensor data. Reference data about the resources being studied are used when and where available to assist the data analysis with the aid of reference data, the analyst extracts information about the type, extent, location and conditions of various resources over which the sensor data were collected. The information is then compile generally in the form of hard copies, maps and tables or as computer files that can be merged with other layers of information in geographical information system. Finally the information is presented to users who apply it to their decision making process.

\section{RESULTS AND DISCUSSION}

By overlay analysis of various thematic maps, it is inferred that certain pockets adjoining to favorable geomorphic zones like buried pediments, valley fills, and buried channels are feasible for further ground water development.

1. The various thematic maps were generated by using Arc view GIS software. By overlay analysis of thematic maps, ground water potential and recharge zonation maps were generated for Gadilam lower sub basin and described in Figure 2.

2. Waste land in each village was identified. Recommendations were also suggested and shown in Figure 3.

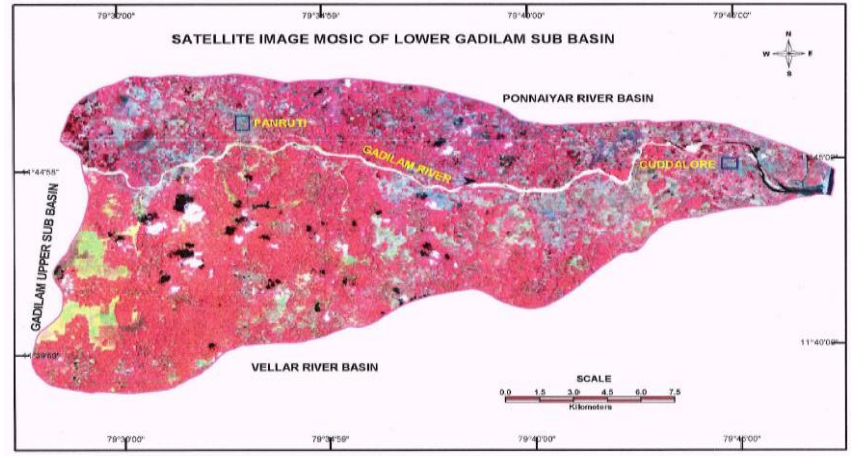

Figure 2. Satellite image of study area

Various thematic maps namely lineament map, land use map, geomorphologic map were prepared from the encoded IRS satellite imageries. Size, shape, scale, shadow, pattern, tone, texture, location and association are important interpretation keys used for identifying different features.IRS-1D-LISS III January 2003 and IRS-1C-LISS-III April 2002 satellite imageries were used for our interpretation.

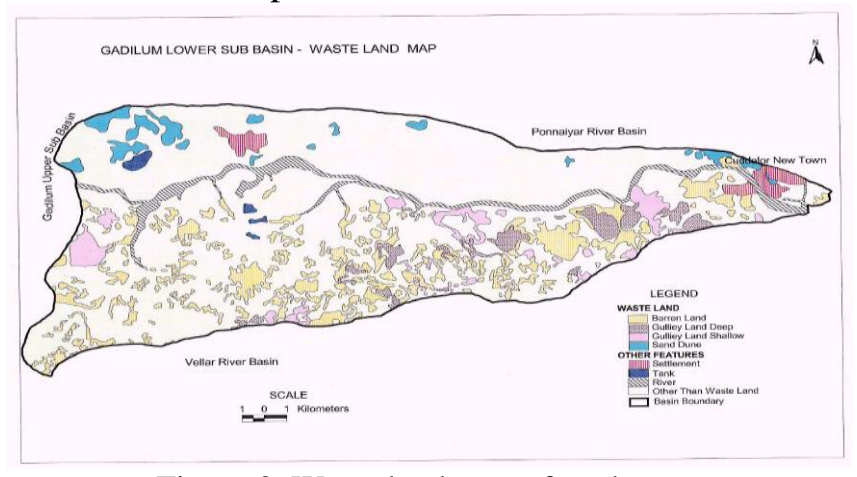

Figure 3. Waste land map of study area

The studied results showing the remedial measure, rain water harvesting and artificial recharge structures are proposed based on Remote sensing studies in favorable geomorphic zones. For this purpose waste land identification and recharge zonation maps were generated by overlay analysis using Arc View GIS software. For increasing the ground water potential for each village different types of artificial recharge structures suitable for the location are recommended. In addition waste lands in each village were identified and the extent \&type of waste lands were furnished. 
For each type of waste land, suggestions are given in the action plan to convert them into productive agricultural lands.

The problem envisaged in the Gadilam lower sub basin,

1. Ground water conservation and reclamation.

2. Droughts.

3. Desiltation in tanks.

4. Socio economic issues.

5. Public awareness and participation.

\section{Conclusion}

The Gadilam lower sub basin lies at southern West part of Villupuram district adjoins Cuddalore district. The study area consists of 3 blocks 205 revenue villages. The administrative area of this area is $335.78 \mathrm{sq} . \mathrm{km}$. The total cultivable area in this area is $205.80 \mathrm{sqkm}$ and it is about $61.19 \%$ of the total administrative area. About $22.38 \%$ of the total area occupied by waste land in this Gadilam lower sub basin which needs priority\& development.

To achieve the objective of identifying favorable areas for further waste land development with in this area following studies were carried out using Remote sensing and GIS techniques.

1. Geological map of the area was generated. The rock type's encountered and favorable geological formation for the identification of waste land.

2. Lineaments their magnitude and direction were deciphered to locate favorable structures for waste land development .Lineament zones were integrated with geomorphic units to identify ground water potential areas.

\section{Scope of the Future Development}

By converting the waste land (land with scrub, land without scrub and stony wastes) into productive agriculture land into possible to enhance the ground water storage for further development.

Most of the waste land areas are covered with stony waste and barren rocky. These lands are left as runoff zones resulting in the sparse vegetation of thorny bushes here and there. For developing the land contour bunding is suggested after clearing the stony waste. Further different types of vegetation cover may be developed to improve this waste land depending of ground water source from weathered/lineament intersection zones.

In addition the following measures may be taken up to improve and modify the waste land area to form a productive area. Wherever the gullies are present, gully plugging is a must to arrest further erosion of soil and conserve the land for utilization. Check dams can also be constructed for effective impounding of runoff water at the favorable locations.

Lineament and lineament intersection zones can be explored for ground water development to supplement water sources. According to surrounding eco-system and environment, suitability of the land, soil cover and water availability; the planning may be made to select suitable horticultural activities for the development of waste lands into cultivation land in the study area. Less water consuming fuel wood trees like citrus, mango, neem tree and other plants may also be planted in the land with or without scrubland category.

\section{References}

[1]. Hongxing, L. Principle of Geographical Information System, Texas A. \& M. University, USA, p.390.

[2]. NWDB.1987.National Waste land Development Board, Description, classification, identification and mapping of waste lands, New Delhi; Ministry of Environmental and Forest, Government of India.

[3]. NRSA 1987, Manual of procedure for waste land mapping using remote sensing techniques, National Remote Sensing Agency.

[4]. Padmini Pani, Mohapatra. 2001. Delineation and monitoring of gullied and ravinces land in part of lower Chambal valley, India using Remote sensing and GIS.22nd Asian conference of Remote sensing, Singapore, pp. $1-5$.

[5]. F503 GIS: Course Notes, School of Forestry, University of Montana, Missoula.

[6]. Gupta et al. Application of remote sensing in inventorying degraded lands using IRS -1A LISS II data. (1996).

[7]. Nagarathinam. Remote sensing and GIS based approach to assess land potential for waste land development around Kalluthu village, Usilampatti block of Tamilnadu, India. (1997).

[8]. Pradeep Kumar. Integrated various thematic layers to understand their influences on waste lands and also explained the utility of remote sensing and GIS techniques for developing them. (2001).

[9]. ESRI Training Manual for Arcview spatial analyst.

[10]. Pushpavathi, K.N., Basavarappa, H.T. 2009. Remote Sensing and GIS Applications for Waste Land Identification. A Case Study in Kollegal Taluk, Chamarajanagar District, Karnataka, India. Journal of Environmental chemistry, 12 (1\& 2), 13-18

[11]. Integrated Mission for Sustainable Development-Technical Guidelines, NRSA, December 1995. 